
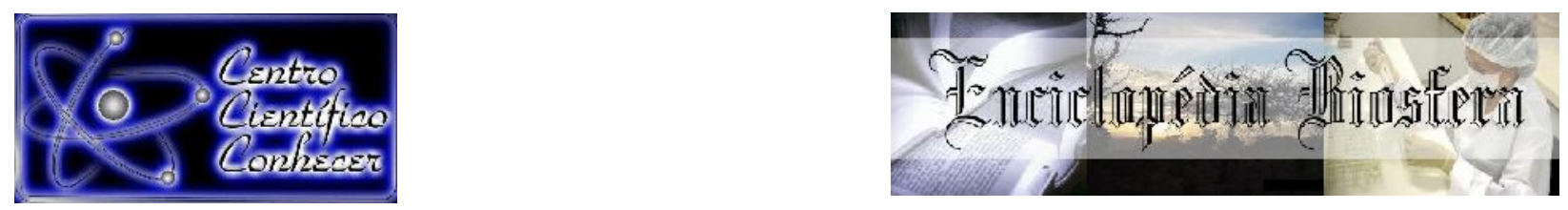

\title{
ANÁLISE DA FREQUÊNCIA DE CONSUMO DE ALIMENTOS POR ALUNOS NADADORES E SEDENTÁRIOS DE TRÊS UNIDADES ESCOLARES DE UMA REDE PRIVADA DO RIO DE JANEIRO
}

\author{
Bruno Castro ${ }^{1}$, Rafael Valladão ${ }^{2}$, Luciano Alonso Valente dos Santos ${ }^{3}$, José Robson \\ de Almeida ${ }^{4}$ \\ ${ }^{1}$ Doutor em Educação Física pela Escola de Educação Física e Desportos da Universidade \\ Federal do Rio de Janeiro (EEFD/UFRJ). \\ 2 Doutorando em Educação pelo Programa de Pós-graduação em Educação da \\ Universidade do Estado do Rio de Janeiro (ProPEd/UERJ). Agência de fomento: CAPES. \\ ${ }^{3}$ Doutor em Ciências da Saúde pela Universidade Federal do Rio Grande do Norte (UFRN). \\ ${ }^{4}$ Doutorando em Educação pela Universidade Lusófona de Humanidades e Tecnologias \\ (CeiED). \\ E-mail: rafaelvalladao1@gmail.com
}

Recebido em: 15/05/2021 - Aprovado em: 15/06/2021 - Publicado em: 30/06/2021 DOI: 10.18677/EnciBio_2021B6

\begin{abstract}
RESUMO
Estudos epidemiológicos têm demonstrado que adolescentes que praticam esportes tem hábitos alimentares mais saudáveis com maior ingestão de frutas e vegetais, enquanto que lanches altamente calóricos estão associados aos sedentários. A pesquisa teve como objetivo analisar e comparar a frequência de consumo de alimentos marcadores de alimentação saudável e não saudável, de alunos nadadores e sedentários de três unidades escolares de uma rede privada de ensino. Foi aplicado questionário para estudantes de $5^{\circ}$ ano do ensino fundamental ao $1^{\circ}$ ano do ensino médio, em três unidades escolares, localizadas nos bairros Taquara e Cachambi no município do Rio de Janeiro e no bairro Mutuá, município de São Gonçalo. O grupo de nadadores foi composto por 29 alunos, que praticam a atividade física "extraclasse" de natação. O grupo controle foi composto por 28 alunos sedentários que não praticavam nenhuma atividade. Os nadadores têm uma maior frequência no consumo de "Frutas frescas e salada de frutas" em relação aos sedentários $(65,5 \%$ vs. $32,1 \%$ sedentários, $(p)=0,01)$. Por ter ocorrido uma maior ingestão de frutas por parte dos nadadores é possível apontar para o entendimento de que o esporte é capaz de incentivar a busca por uma alimentação mais saudável.
\end{abstract}

PALAVRAS-CHAVE: Alimentação; Educação; Natação.

\section{ANALYSIS OF THE FREQUENCY OF FOOD CONSUMPTION BY SWIMMING AND SEDENTARY STUDENTS FROM THREE SCHOOL UNITS IN A PRIVATE NETWORK IN RIO DE JANEIRO}

\begin{abstract}
Epidemiological studies have shown that adolescents who play sports have healthier eating habits with a greater intake of fruits and vegetables, while high-calorie snacks are associated with sedentary ones. The research aimed to analyze and compare the frequency of consumption of foods that are indicators of healthy and unhealthy food, of swimming and sedentary students from three school units in a private school
\end{abstract}


system. A questionnaire was applied to students from the 5th grade of elementary school to the 1st year of high school, in three school units, located in the neighborhoods Taquara and Cachambi in the municipality of Rio de Janeiro and in the neighborhood Mutuá, municipality of São Gonçalo. The group of swimmers was composed of 29 students, who practice "extra-class" swimming physical activity. The control group was composed of 28 sedentary students who did not practice any activity. Swimmers have a higher frequency of consumption of "Fresh fruits and fruit salad" in relation to sedentary ones $(65.5 \%$ vs. $32.1 \%$ sedentary, $(p)=0.01)$. Because there was a greater intake of fruit by swimmers, it is possible to point to the understanding that sport is able to encourage the search for a healthier diet.

KEYWORDS: Food; Education; Swimming.

\section{INTRODUÇÃO}

O período da adolescência torna-se crítico uma vez que ocorre a incidência da obesidade em decorrência da predominância de atividades sedentárias de lazer e práticas alimentares não-saudáveis que são objeto de estudo e investigações em diversas pesquisas, em países desenvolvidos e em desenvolvimento. Estas práticas repercutem na saúde desta população infanto-juvenil (ÁVILA et al., 2019). Tais hábitos criados na infância e adolescência são comportamentos de risco cada vez mais frequentes e serão levados para a fase adulta, podendo ocasionar doenças crônicas (COSTA et al., 2018; MONTEIRO et al., 2020).

A título de exemplos pode-se citar: o consumo de lanches hipercalóricos que substituem as principais refeições diárias (café da manhã, almoço e jantar), a ingestão elevada de alimentos ricos em carboidratos refinados, gorduras saturadas, fast food, açúcar, alimentos de alta densidade energética, alto consumo de refrigerantes, reduzindo consumo de marcadores de alimentação saudável, como laticínios, hortaliças e vegetais (VALLIN et al., 2020). Há também fatores de riscos relacionados à saúde quando foi observado o estilo de vida de adolescentes no que tange ao baixo consumo de frutas, a inatividade física, a falta de habilidade em manter o peso corporal (MONTEIRO et al., 2020).

De acordo com Costa et al. (2018), estudos nacionais e internacionais identificaram que nessa faixa etária os hábitos alimentares não-saudáveis estão associados a classes econômicas com maior poder aquisitivo e maior acesso à informação, ocorrendo maior consumo de gorduras, açúcares, sódio e a baixa ingestão de hortaliças. A maior ingestão de grãos como o arroz e feijão entre os adolescentes estão associados aos de famílias mais pobres (COSTA et al., 2018). A respeito do consumo não-saudável de alimentos, em relação a ambos os gêneros, Fernandes et al. (2011), concluíram que mais de $80 \%$ dos adolescentes ingerem pouca quantidade de fibras e aproximadamente $35 \%$ ingerem grande quantidade de gordura.

A promoção de hábitos saudáveis deve ser tomada como prioritária por todos os setores da sociedade civil (MONTEIRO et al., 2020). Nesse sentido a escola tem papel primordial no desenvolvimento desses atos de conscientização e promoção de saúde, uma vez que pode promover ações de melhorias do estado nutricional dos educandos, contribuindo para o desenvolvimento humano (ÁVILA et al., 2019; MONTEIRO et al., 2020). A escola deve promover saúde junto com a formação cidadã, estimulando o exercício de direitos e deveres, qualidade de vida, atitudes e comportamentos saudáveis a todos os integrantes da comunidade escolar, tendo estes como tema de seu projeto político pedagógico (CAMOZZI et al., 2015). 
Outro dado que não pode ser ignorado é que, em média, 2,8 milhões de pessoas morrem no mundo, por ano, em decorrência do sobrepeso ou obesidade (WHO, 2020). Apesar das recomendações da Organização Mundial da Saúde (OMS) a respeito da atividade física que estipula 60 minutos ou mais de atividade física diárias moderada a vigorosa (WHO, 2018). Em relação aos hábitos alimentares mais saudáveis dos adolescentes, estudos epidemiológicos mostram que estes estão associados aos que praticam esportes e que a menor ingestão de frutas, vegetais e maior lanches altamente calóricos são observadas nos sedentários. Também ocorre um maior tempo assistindo televisão, jogando vídeo game, utilizando o celular, tablet e computador, ultrapassando o limite de duas horas diárias para se gastar com lazer sedentário, proposto pela Academia Americana de Pediatria (COELHO et al., 2016; FERNANDES et al., 2011).

Neste contexto, o objetivo do presente estudo foi analisar e comparar os hábitos alimentares, de alunos nadadores e sedentários de três unidades escolares de uma rede privada do Rio de Janeiro, levantando a hipótese de que os nadadores têm hábitos alimentares mais saudáveis.

\section{MATERIAL E MÉTODOS}

A pesquisa foi desenvolvida no segundo semestre do ano de 2019, com estudantes de $5^{\circ}$ ano do ensino fundamental ao $1^{\circ}$ ano do ensino médio, em três unidades escolares de uma rede privada do Rio de Janeiro, localizadas nos bairros Taquara e Cachambi no Rio de Janeiro-RJ e no bairro Mutuá em São Gonçalo-RJ. Os pesquisadores colheram autorização dos pais ou responsáveis, por se tratar de alunos menores de idade. O grupo de nadadores foi composto por 29 alunos ( Taquara $=10$, Cachambi $=7$, São Gonçalo $=12$ ), que praticam a atividade física "extraclasse" de natação, no contraturno escolar. Esses alunos, apesar de terem uma rotina que se aproxima de atletas, seus treinos não têm como objetivo o rendimento esportivo e sim a qualidade de vida. O grupo controle foi composto por 28 alunos sedentários (Taquara $=8$, Cachambi $=8$, São Gonçalo $=12$ ), que praticavam somente a Educação Física curricular na escola (somente 50 minutos por semana) e nenhuma outra atividade física fora desta.

Utilizou-se como referência para as classificações: nadadores e sedentários. As recomendações da Organização Mundial da Saúde (OMS), para crianças entre cinco e 17 anos, que estipula 60 minutos ou mais de atividade física diárias moderada a vigorosa (WHO, 2018). Nessa perspectiva, os nadadores se aproximam dessas recomendações com os treinos de natação que tem duração média de uma hora e meia, ocorrendo durante quatro dias por semana, além da Educação Física curricular de 50 minutos. Enquanto os sedentários somente praticam as aulas de Educação Física curricular, como supramencionado.

Os dados referentes às práticas alimentares dos nadadores e sedentários foram coletados pela aplicação de questionário com questões fechadas. Os pesquisadores foram até as unidades escolares, em que os estudantes mantêm suas aulas de natação e estudam, para realizar a aplicação. As questões incluíram hábito e frequência semanal em que realizavam café da manhã, almoço (comida ou lanche), jantar (comida ou lanche) e consumiam marcadores de alimentação saudável tais como feijão, verduras e legumes, frutas frescas ou saladas de frutas, e marcadores de alimentação não saudável tais como salgados fritos, guloseimas (doces, balas, tortas), refrigerante, alimentos processados, biscoitos doces ou salgados, bebidas diversas (sucos de caixinha, guaraná natural, achocolatados) e fast food. Além de também verificar a frequência semanal de consumo de lanches 
trazidos de casa para a escola, comprados na cantina da escola ou vendidos por ambulantes.

Os dados foram digitados utilizando-se o software Epidata, versão XX. Para as análises estatísticas foi utilizado o software IBM SPSS Statistics 22. Os grupos de nadadores e sedentários foram comparados quanto à frequência de consumo dos marcadores de alimentação saudável e não saudável pelo teste Qui quadrado, considerando-se o valor de $p<0,05$ para significância estatística.

Considerou-se "menor frequência no consumo dos alimentos" ou de prática de refeições quando a resposta ao questionário era para o consumo de uma a duas vezes por semana ou nunca. A "maior frequência" de três a quatro vezes na semana; cinco a seis vezes na semana e diariamente, referente aos últimos sete dias que precederam a avaliação.

O projeto foi aprovado pelo Comitê de Ética em Pesquisa do Instituto de Psiquiatria (IPUB) da Universidade Federal do Rio de Janeiro (UFRJ), em três de julho de 2019, processo nำ 11249919.1.0000.5263.

\section{RESULTADOS E DISCUSSÃO}

Foram avaliados 57 adolescentes, sendo 28 sedentários e 29 nadadores. 0 Quadro 1 demonstra que a média de minutos de atividade física semanal dos nadadores (360 minutos) é muito superior à dos sedentários (60 minutos), e que o tempo de permanência na escola é maior em relação aos nadadores ( $9 \mathrm{~h} v s$. $5 \mathrm{~h}$ ).

QUADRO 1. Comparação entre a rotina dos alunos nadadores e sedentários.

\begin{tabular}{|l|c|c|}
\hline VARIÁVEL / GRUPO & NADADORES & SEDENTÁRIOS \\
\hline $\begin{array}{l}\text { Média de atividade física } \\
\text { semanal }\end{array}$ & 410 minutos & 50 minutos \\
\hline $\begin{array}{l}\text { Média de tempo de } \\
\text { permanência na escola (por } \\
\text { dia) }\end{array}$ & 9 horas & 5 horas \\
\hline
\end{tabular}

Quanto às refeições, na Tabela 1 pode-se observar que os nadadores têm uma maior frequência no consumo de Café da manhã $(82,8 \%$ vs. $60,7 \%)$, Almoço/comida (100,0\% vs. 92,9\%) e Jantar/lanche (31,0\% vs. 14,3\%). Enquanto os sedentários têm maior frequência no consumo de Almoço/lanche (10,7\% vs. 3,4\%) e Jantar/comida $(96,4 \%$ vs. 86,2\%). Apesar de não haver diferença estatística relevante entre os grupos, ainda assim é importante colocar que a substituição de refeição principal por lanche é mais comum entre os sedentários. De acordo com Vallin et al. (2020), os lanches rápidos estão entre a preferência dos adolescentes para substituir refeições. 
TABELA 1. Frequência de refeições de alunos nadadores e sedentários.

\begin{tabular}{|c|c|c|c|c|c|}
\hline & \multicolumn{3}{|c|}{ SEDENTÁRIOS } & \multirow{2}{*}{$\begin{array}{c}\text { NADADORES } \\
\%\end{array}$} & \multirow[t]{2}{*}{ Valor de $p^{1}$} \\
\hline & $\mathbf{n}^{\star}$ & $\%$ & $\mathbf{n}^{*}$ & & \\
\hline Café da manhã & 28 & & 29 & & 0,06 \\
\hline MENOR FREQUÊNCIA ${ }^{2}$ & & 39,3 & & 17,2 & \\
\hline MAIOR FREQUÊNCIA ${ }^{3}$ & & 60,7 & & 82,8 & \\
\hline Almoço (comida) & 28 & & 29 & & 0,14 \\
\hline MENOR FREQUÊNCIA ${ }^{2}$ & & 7,1 & & 0,0 & \\
\hline MAIOR FREQUÊNCIA ${ }^{3}$ & & 92,9 & & 100,0 & \\
\hline Almoço (lanche) & 28 & & 29 & & 0,28 \\
\hline MENOR FREQUÊNCIA ${ }^{2}$ & & 89,3 & & 96,6 & \\
\hline MAIOR FREQUÊNCIA ${ }^{3}$ & & 10,7 & & 3,4 & \\
\hline Jantar (comida) & 28 & & 29 & & 0,17 \\
\hline MENOR FREQUÊNCIA ${ }^{2}$ & & 3,6 & & 13,8 & \\
\hline MAIOR FREQUÊNCIA ${ }^{3}$ & & 96,4 & & 86,2 & \\
\hline Jantar (lanche) & 28 & & 29 & & 0,13 \\
\hline MENOR FREQUÊNCIA ${ }^{2}$ & & 85,7 & & 69,0 & \\
\hline MAIOR FREQUÊNCIA ${ }^{3}$ & & 14,3 & & 31,0 & \\
\hline
\end{tabular}

$\mathrm{Na}$ Tabela 2 pode-se observar que os legumes, verduras e frutas, alimentos que estão entre os considerados mais saudáveis, para Mendes e Catão (2010) e por uma vasta gama de pesquisadores da área da nutrição, tem maior frequência de consumo entre os nadadores. 
TABELA 2. Frequência de alimentos ingeridos por alunos nadadores e sedentários, em sete dias da semana.

\begin{tabular}{|c|c|c|c|c|c|}
\hline & \multicolumn{3}{|c|}{ SEDENTÁRIO } & \multirow{2}{*}{$\begin{array}{c}\text { NADADOR } \\
\%\end{array}$} & \multirow[t]{2}{*}{ Valor de $p^{1}$} \\
\hline & $\mathrm{n}^{*}$ & $\%$ & $\mathrm{n}^{*}$ & & \\
\hline Feijão & 28 & & 29 & & 0,52 \\
\hline MENOR FREQUÊNCIA 2 & & 14,3 & & 20,7 & \\
\hline MAIOR FREQUÊNCIA ${ }^{3}$ & & 85,7 & & 79,3 & \\
\hline Salgados fritos & 28 & & 29 & & 0,47 \\
\hline MENOR FREQUÊNCIA ${ }^{2}$ & & 89,3 & & 82,8 & \\
\hline MAIOR FREQUÊNCIA ${ }^{3}$ & & 10,7 & & 17,2 & \\
\hline Legume ou verdura & 28 & & 29 & & 0,08 \\
\hline MENOR FREQUÊNCIA ${ }^{2}$ & & 60,7 & & 37,9 & \\
\hline MAIOR FREQUÊNCIA ${ }^{3}$ & & 39,3 & & 62,1 & \\
\hline Guloseimas & 28 & & 29 & & 0,50 \\
\hline MENOR FREQUÊNCIA ${ }^{2}$ & & 46,4 & & 55,2 & \\
\hline MAIOR FREQUÊNCIA ${ }^{3}$ & & 53,6 & & 44,8 & \\
\hline $\begin{array}{l}\text { Frutas frescas ou } \\
\text { salada de frutas }\end{array}$ & 28 & & 29 & & 0,01 \\
\hline MENOR FREQUÊNCIA ${ }^{2}$ & & 67,9 & & 34,5 & \\
\hline MAIOR FREQUÊNCIA ${ }^{3}$ & & 32,1 & & 65,5 & \\
\hline Refrigerante & 28 & & 29 & & 0,15 \\
\hline MENOR FREQUÊNCIA ${ }^{2}$ & & 82,1 & & 65,5 & \\
\hline MAIOR FREQUÊNCIA ${ }^{3}$ & & 17,9 & & 34,5 & \\
\hline $\begin{array}{l}\text { Alimentos ultra } \\
\text { processados }\end{array}$ & 28 & & 29 & & 0,13 \\
\hline MENOR FREQUÊNCIA ${ }^{2}$ & & 57,1 & & 75,9 & \\
\hline MAIOR FREQUÊNCIA ${ }^{3}$ & & 42,9 & & 24,1 & \\
\hline $\begin{array}{l}\text { Biscoitos doces ou } \\
\text { salgados }\end{array}$ & 28 & & 29 & & 0,89 \\
\hline MENOR FREQUÊNCIA ${ }^{2}$ & & 50,0 & & 48,3 & \\
\hline MAIOR FREQUÊNCIA ${ }^{3}$ & & 50,0 & & 51,7 & \\
\hline
\end{tabular}




\begin{tabular}{|c|c|c|c|c|c|}
\hline & & ENTÁ & & NADADOR & Valor de $p^{1}$ \\
\hline & $\mathbf{n}^{*}$ & $\%$ & $\mathbf{n}^{\star}$ & $\%$ & \\
\hline Bebidas diversas & 28 & & 29 & & 0,35 \\
\hline MENOR FREQUÊNCIA ${ }^{2}$ & & 46,4 & & 58,6 & \\
\hline MAIOR FREQUÊNCIA ${ }^{3}$ & & 53,6 & & 41,4 & \\
\hline $\begin{array}{l}\text { Lanche tipo "Fast } \\
\text { food" }\end{array}$ & 28 & & 29 & & 0,57 \\
\hline MENOR FREQUÊNCIA ${ }^{2}$ & & 96,4 & & 93,1 & \\
\hline MAIOR FREQUÊNCIA ${ }^{3}$ & & 3,6 & & 6,9 & \\
\hline Lanche trazido de casa & 28 & & 29 & & 0,21 \\
\hline MENOR FREQUÊNCIA ${ }^{2}$ & & 67,9 & & 51,7 & \\
\hline MAIOR FREQUÊNCIA 3 & & 32,1 & & 48,3 & \\
\hline $\begin{array}{l}\text { Lanche comprado na } \\
\text { cantina }\end{array}$ & 28 & & 29 & & 0,24 \\
\hline MENOR FREQUÊNCIA ${ }^{2}$ & & 82,1 & & 69,0 & \\
\hline MAIOR FREQUÊNCIA ${ }^{3}$ & & 17,9 & & 31,0 & \\
\hline $\begin{array}{l}\text { Lanche de vendedor } \\
\text { ambulante }\end{array}$ & 28 & & 29 & & 0,30 \\
\hline MENOR FREQUÊNCIA ${ }^{2}$ & & 96,4 & & 100,0 & \\
\hline MAIOR FREQUÊNCIA ${ }^{3}$ & & 3,6 & & 0,0 & \\
\hline
\end{tabular}

A variável: "Legume ou verdura" não apresentou diferença estatística significativa entre os grupos, mas maior frequência de consumo entre os nadadores (nadadores $62,1 \%$ vs. $39,3 \%$ sedentários). As demais variáveis presentes na Tabela 3 também não. Porém a variável "Frutas frescas e salada de frutas" (nadadores $65,5 \%$ vs. $32,1 \%$ sedentários) sim, como supracitado. Tal dado vem confirmar algo que é consenso no meio científico e em diversas pesquisas da área: a prática esportiva está associada à busca por uma alimentação mais saudável. No entanto, há de se pontuar que não se pode ignorar que no consumo dos alimentos não saudáveis não há diferença entre os grupos, ou seja, nadadores e não nadadores comem alimentos não saudáveis como todos os adolescentes. Por exemplo, nadadores consomem muito mais refrigerantes que sedentários, como se pode observar na Tabela 2.

Há de se ressaltar também que os alunos nadadores também possuem o hábito de almoçar no refeitório da escola, que tem cardápio elaborado por nutricionista, e/ou trazer o almoço de casa para consumir na escola, constituindo ENCICLOPÉDIA BIOSFERA, Centro Científico Conhecer - Jandaia-GO, v.18 n.36; p. 95 2021 
assim uma rotina de estudos, alimentação e treinos de natação, em uma perspectiva de educação semi-integral. Já os sedentários estudam somente um turno (manhã ou tarde) e após retornam para suas casas.

\section{CONCLUSÃO}

O sedentarismo ainda é uma realidade presente no cotidiano de diversas crianças e adolescentes, mas tem na escola o enfrentamento necessário na figura do professor de Educação Física. A rede privada de ensino pesquisada tem essa preocupação e esse entendimento, e vem buscando, ao longo dos anos, promover a conciliação entre o rendimento acadêmico, a alimentação saudável e uma rotina esportiva.

Os resultados inconclusivos, com as diferenças estatísticas não-significativas no que concerne à frequência de ingestão e tipos de alimentos consumidos, pode ser explicado devido ao baixo número de nadadores pesquisados e o grupo controle de sedentários equivalentes em faixa etária. Sugere-se que as escolas incentivem as atividades físicas bem como introdução de educação alimentar, pois gerariam dados ainda mais relevantes sobre a qualidade da alimentação em pesquisas futuras.

No entanto, pode-se concluir também que por ter ocorrido maior ingestão de frutas por parte dos nadadores é possível apontar para o entendimento de que o esporte nas escolas pesquisadas pode ser capaz de incentivar a busca por uma alimentação mais saudável. Os alunos sedentários estão mais suscetíveis a alimentação não saudável por ter uma rotina escolar mais flexível que os nadadores, uma vez que não têm carga horária de atividade física a ser cumprida semanalmente. Recomenda-se um estudo mais aprofundado acerca da alimentação dos alunos, não só no Rio de Janeiro, mas em nível nacional e que o sedentarismo não seja somente objeto de pesquisa, mas sim um desafio a ser vencido nas escolas brasileiras.

\section{REFERÊNCIAS}

ÁVILA, R. S. de; PANSERA, D. V. do C.; PREVIDENTE, N. Z.; ROSSAROLLA, T. da S.; BACKES, V. Educação alimentar e nutricional para adolescentes: O protagonismo como estratégia para práticas alimentares saudáveis. Revista Saúde e Desenvolvimento Humano, Canoas, v. 7, n. 3, p. 39-48, 2019. Disponível em $<$ https://revistas.unilasalle.edu.br/index.php/saude_desenvolvimento/article/view/505 3>. doi: $10.18316 /$ sdh.v7i3.5053

CAMOZZI, A. B. Q.; MONEGO, E. T.; MENEZES, I. H. C. F.; SILVA, P. O. Promoção da Alimentação Saudável na Escola: realidade ou utopia?. Cadernos de Saúde Coletiva, Rio de Janeiro, n. 23 v. 1, p. 32-7, 2015 . Disponível em: $<$ https://www.scielo.br/pdf/cadsc/v23n1/1414-462X-cadsc-23-01-00032.pdf>. doi:10.1590/1414-462X201500010006

COELHO, L. F.; SIQUEIRA, J. H.; MOLINA, M. del C. B. Estado nutricional, atividade física e tempo de tela em escolares de 7-10 anos: um estudo de intervenção em Vitória-ES. Demetra: Alimentação, Nutrição \& Saúde, n. 11 v. 4, p. 1067-1083, 2016. Disponível em: < https://www.epublicacoes.uerj.br/index.php/demetra/article/view/22030>. doi: 10.12957/demetra.2016.22030 
FERNANDES, R. A.; CHRISTOFARO, D. G. D.; CASONATTO, J.; KAWAGUTI, S. S.; RONQUE, E. R. V.; CARDOSO, J. R.; FREITAS JÚNIOR, I. F.; OLIVEIRA, A. R. Associação transversal entre hábitos alimentares saudáveis e não saudáveis e atividade física de lazer em adolescentes. Jornal de Pediatria. n. 3, v. 87, 2011. Disponível em: <https://www.scielo.br/pdf/jped/v87n3/a12v87n03.pdf>. doi: 10.2223/JPED.2093

COSTA, C. dos S.; FLORES, T. R.; WENDT, A.; NEVES, R. G.; ASSUNÇÃO, M. C. F.; SANTOS, I. S. Comportamento sedentário e consumo de alimentos ultraprocessados entre adolescentes brasileiros: Pesquisa Nacional de Saúde do Escolar (PeNSE), 2015. Cadernos de Saúde Pública, n. 34, v. 3, p. 1-12, 2018. Disponível em: <https://www.scielo.br/pdf/csp/v34n3/1678-4464-csp-34-03e00021017.pdf > doi: 10.1590/0102-311x00021017

MONTEIRO, L. Z.; VARELA, A. R.; SOUZA, P. de; MANIÇOBA, A. C. M.; BRAGA JÚNIOR; F. Hábitos alimentares, atividade física e comportamento sedentário entre escolares brasileiros: Pesquisa Nacional de Saúde do Escolar, 2015. Revista Brasileira de Epidemiologia, Rio de Janeiro, v. 23, 2020. Disponível em: <https://www.scielo.br/pdf/rbepid/v23/1980-5497-rbepid-23-e200034.pdf> doi: 10.1590/1980-549720200034

MENDES, K. L.; CATÃO, L. P. Avaliação do consumo de frutas, legumes e verduras por adolescentes de formiga - MG e sua relação com fatores socioeconômicos. Alimentos e Nutrição, Araraquara, v. 21, n. 2, p. 291-296, abr./jun. 2010. Disponível em:

<https://www.researchgate.net/publication/49600243_AVALIACAO_DO_CONSUMO _DE_FRUTAS_LEGUMES_E_VERDURAS_POR_ADOLESCENTESS_DE_FORMIG A -

MG E SUA RELACAO COM FATORES SOCIOECONOMICOS/fulltext/00b1010 70cf245659d01129b/AVALIIACAŌ-DO-CONSUMO-DE-FRUTAS-LEGUMES-EVERDURAS-POR-ADOLESCENTES-DE-FORMIGA-MG-E-SUA-RELACAO-COMFATORES-SOCIOECONOMICOS.pdf>

VALLIN, C.; NAVES, L. M.; OLIVEIRA, R. C. de; GOMES, J. P. de O. Alimentação saudável na adolescência. Revista de Educação Popular, v. 19, n. 3, p. 193-209, 23 nov. 2020. Disponível em: <http://www.seer.ufu.br/index.php/reveducpop/article/view/53181> doi: 10.14393/REP-2020-53181

WHO - World Health Organization. More active people for a healthier world: Global action plan on physical activity 2018-2030: more active people for a healthier world. Geneva: World Health Organization, 2018. Disponível em: <https://www.who.int/ncds/prevention/physical-activity/global-action-plan-2018-

2030/en/>

WHO - World Health Organization. Obesity: World Health Organization, 2020. Disponível em: <http://www.who.int/features/factfiles/obesity/en/> 